\title{
A CALL CENTER ORIENTED CONSULTANT SYSTEM FOR FISH DISEASE DIAGNOSIS IN CHINA
}

\author{
Jie Zhang ${ }^{1,2}$, Daoliang $\mathrm{Li}^{1,2, *}$ \\ ${ }^{1}$ China Agricultural University, College of Information and Electrical Engineering, Beijing \\ 100083, China \\ ${ }^{2}$ Key Laboratory of Modern Agriculture System Integration, China Agricultural University, \\ Beijing 100083, China \\ "Corresponding Author, Tel: +86-10-62736717, Fax: +86-10-62737679, Email: \\ dliangl@cau.edu.cn or
}

Abstract: $\quad$ Fish expert systems have been developed to solve the fish disease diagnosis problems. However, despite these inherent advantages, there are some limitations yet in the application of the systems because of the poor infrastructure and low capacity for the use of computers. A Call Center-based consultant system for fish disease diagnosis has been developed to overcome the difficulties, and the objective of this system is the automation of fish diagnostic process based on call center via the telephone. This paper explains the needs for the consultant system, the fish disease diagnosis process and the development of the system. The system structure and its components are described. It can provide the accurate and timely diagnosis, prevention and treatment all around the clock and everywhere the fish sites are located. The experience and the further development of the system are also discussed in the paper.

Keywords: Call Center, fish disease diagnosis, consultant system, knowledge acquisition

\section{INTRODUCTION}

Great efforts have been put by researchers, institutes and organizations to solve the fish disease diagnosis problems in China. Especially, a number of 
agricultural web sites have emerged on the internet providing on-line information in the form of Hyper Text Markup Language (HTML) pages. (Allan et al., 2000) A web-based expert system for fish disease diagnosis, called Fish-Expert, has been developed by Agricultural Information Technology Institute at China Agricultural University (Daoliang et al., 2002).

As most aquaculture sites are scattered in remote rural areas which are weak in infrastructure and capability for the use of computers. Fish farmers can hardly get the service the Fish-Expert provides and the application of the system is restricted to some extent. Nowadays, with the rapid development of Telecommunication Industry, the Telephone Coverage Rate has been increased in Rural Areas in China. Especially, after the Cun Cun Tong program was launched. In early 2004, telephone services were available in 630,000 villages, or around 91 percent of the nation's total. By November 2005 the figure had been raised to 97 percent (http://english.sohu.com /20060314/n227789404.shtml).

Agriculture distance diagnosis has been applied since the 1990s (Holmes et al., 2000). A call center can provide telephone access to information services that are delivered by a human operator (agent), who in turn has access to an information resource (database) (Marco et al., 2004), or by IVR.

This paper presents a call center-based consultant system for fish disease diagnosis. It was a major outcome of Huo Ying Dong Fundation (Contract Number 94032). During the development of the system, fish expert system and the call center were integrated together to overcome the limitations and enhance the functionality of traditional ES. The system can provide the accurate and timely diagnosis, prevention and treatment all around the clock and everywhere the fish sites are located via the telephone. It has been tested and evaluated in Tianjin province of North China. The experience with developing the system, lessons learnt and conclusions are provided at the end of the paper.

\section{SYSTEM DESIGN AND DEVELOPMENT}

Though rural areas are weak in the infrastructure and capacity for the use of computers, telephone and mobile uses increase rapidly in rural China. And also the provision of call centre service appears as a sound alternative support channel for fish farmer to acquire counseling and support in disease diagnosis in China. So a call center-based consultant system for fish disease diagnosis was developed that allows fish farmers to easily get the fish disease diagnosis information via the telephone. 


\subsection{System architecture}

The overall architecture of the system contains three parts. Users including mobile phone users and telephone users are connected to the call center via the telephone. Then calls are distributed to the IVR (Interactive Voice Response) or the Agents by the ACD (Automatic call distribution). IVR is a technology that automates interaction with telephone callers. IVR solutions use pre-recorded voice prompts and menus to present information and options to fish farmers, and touch-tone telephone keypad entry to gather responses, also helps the caller to be connected to an agent who can assist them. For the fish farmer, he or she can describe the symptoms of their fishes' disease to the call center agent by telephone, and they input all these symptoms into the Expert system for fish disease diagnosis interface, then tell the fish farmers the diagnosis, prevention and the treatment. After many fish farmers using the system, a great quantity of fish disease diagnosis cases were acquired and stored in the case base for the further knowledge acquisition. Some efficient algorithms will be used to find hidden patterns in data and generate sets of decision rules from cases. Then they will be used in the next diagnosis processing.

\subsection{System work flow}

Telephone callers (or wireless callers) dial, typically, a toll-free telephone number to contact the call center via the PSTN (or PLMN), then the interactive voice response (IVR) system prompts the caller with a series of announcements and menus. The caller chooses the service mode from IVR automatic service and agent service by pressing the right number.

For general fish disease problems, the call center is usually "front-ended" with the interactive voice response system. The IVR tree has some main branches (e.g. on-the-sport behavior query, body part query, etc) represented by the telephone number from 1 to N. Each main branch has its own branches corresponding to the phone number. For instance, after you choosing the on-the-sport behavior query, you still should make a choice from the multiple symptoms. And at last the treatment, prevention and the diagnosis will be given to you. Maybe you can not get a conclusion by one symptom, what you should do then is to press \# button for returning to the main menus and choose other symptoms until you get the suggestion.

In some cases, if a caller wants to exit the IVR system at certain points in the "dialogue", he can press any key at any time to disconnect the telephone directly. Callers needn't wait to make a key choice until the end of every performance. The system is allocated to each call only for a very short fraction of time, an IVR experience may have a duration of several minutes. 
Therefore, if time outs, the phone will be disconnected by the system automatically.

After choosing to speak directly with an agent, a caller is routed to the agent that has been idle for the longest duration. Otherwise, if all agents are busy, the caller's call is routed to the queue with the shortest expected wait time. The agents interact with the expert system for fish disease diagnosis that provides the fish disease knowledge.

\subsection{Knowledge acquisition}

Knowledge acquisition is the process of eliciting the expertise of domain expert and of formulating the expertise into a representation that can be used by a knowledge-based system. It is the most difficult stage in the development of expert systems. Many techniques have been developed for knowledge acquisition (KA). Some commonly used approaches are interviews, observations, taking experts through case studies and rule induction by machines.

Plenty of the fish disease diagnosis cases can be required from the call center system, maybe the cases available are insufficient or poorly-specified. The challenge here is to get hold of the information, and turn them into knowledge by making cases usable. A knowledge acquisition tool can be used to produce a set of rule cases with the quantity of the original cases.

In this system, Rough sets theory and neural networks are combined together to acquire knowledge for their complementary features. The rough set theory, proposed by Pawlak in 1982 (Pawlak et al., 1982) adopts the concept of equivalence classes to partition training instances according to some criteria. The rule induction from the original data model is data-driven without any additional assumptions. Neural networks (NN) are a computer 'simulation' of the interconnected neurons in our brains. This method simulates a network of interconnected neurons usually arranged in three layers, where the lowest one receives inputs and the top signals the outputs.

The approach is to use rough set approach as a pre-processing tool for the neural networks. By eliminating the redundant data from database, rough set methods can greatly accelerate the network training time and improve its prediction accuracy. Rough set method was also applied to generating rules from trained neural networks. In the system, neural networks were the main knowledge bases and rough sets were used only as a tool to speedup or simplify the process of using neural networks for mining knowledge from the databases. 


\section{DISCUSSIONS AND CONCLUSIONS}

The system demonstrates the possibility and potential benefits of using the call center to facilitate the expert system in aquaculture. So it is a good idea for integrating call center system with the fish disease diagnosis expert system, the two systems can complement with each other, and meet the needs for the fish farmers and vets in different situations to overcome the limitations of each individual system.

This paper presents the development of a call-center based consultant system for fish disease. This system is a good try for telemedicine techniques application based on the call center in aquaculture. It realized the longdistance fish disease diagnosis without having the necessary IT equipment or low Internet access speed in rural areas in China and was successfully used in the practice. It presents the tendency of the telemedicine techniques application based on call center in Agriculture, we will continue this interesting work unmoved.

\section{ACKNOWLEDGEMENTS}

This research is supported by the Huo Ying Dong Fundation (Contract Number: 94032) in China. We would like to thank many domain experts from the Beijing Aquaculture Science Institute, Aquaculture Department of Tianjin Agricultural College, Aquaculture Bureau of Shandong province, for their co-operation and support. Our special thanks should go to Dr Zahng Xiaoshuan for the fish disease diagnosis and Dr Zhang Jian for the call center use. We would also like to acknowledge Mr YouLongyong and $\mathrm{Mr}$ Zhuwei for their valuable suggestions on the improvement of the system.

\section{REFERENCES}

Allan Leck Jensen, Peter S. Boll, Iver Thysen and B.K. Pathak., PlanteInfor - a web-based system for personalised decision support in crop management, Computers and Electronics in Agriculture, 25 (2000), 271-293.

Daoliang Li, Zetian Fu and Yanqing Duan, Fish-Expert: a web-based expert system for fish disease diagnosis, Expert Systems with Applications, 23 (2002), 311-320.

http://english.sohu.com/20060314/n227789404.shtml

Marco Adria and Shamsud D. Chowdhury, Centralization as a design consideration for the management of call centers, Information and Management, 41 (2004), 497-507.

Pawlak, Z, Rough set, International Journal of Computer and Information Sciences, 341-356, 1982. 